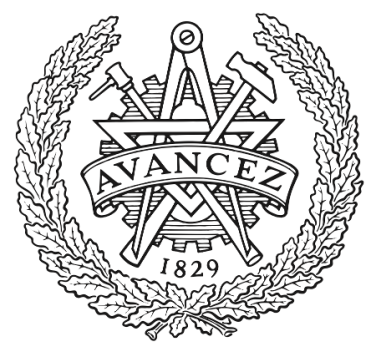

CHALMERS

UNIVERSITY OF TECHNOLOGY

\title{
Cognition as Embodied Morphological Computation
}

Downloaded from: https://research.chalmers.se, 2023-04-26 10:02 UTC

Citation for the original published paper (version of record):

Dodig Crnkovic, G. (2018). Cognition as Embodied Morphological Computation. Studies in Applied Philosophy, Epistemology and Rational Ethics: 19-23.

http://dx.doi.org/10.1007/978-3-319-96448-5_2

N.B. When citing this work, cite the original published paper. 


\title{
Cognition as Embodied Morphological Computation
}

\author{
Gordana Dodig-Crnkovic ${ }^{1,2(-)}$ \\ ${ }^{1}$ Chalmers University of Technology, Gothenburg, Sweden \\ dodig@chalmers.se \\ ${ }^{2}$ Gothenburg University, Gothenburg, Sweden
}

\begin{abstract}
Cognitive science is considered to be the study of mind (consciousness and thought) and intelligence in humans. Under such definition variety of unsolved/unsolvable problems appear. This article argues for a broad understanding of cognition based on empirical results from i.a. natural sciences, selforganization, artificial intelligence and artificial life, network science and neuroscience, that apart from the high level mental activities in humans, includes subsymbolic and sub-conscious processes, such as emotions, recognizes cognition in other living beings as well as extended and distributed/social cognition. The new idea of cognition as complex multiscale phenomenon evolved in living organisms based on bodily structures that process information, linking cognitivists and EEEE (embodied, embedded, enactive, extended) cognition approaches with the idea of morphological computation (info-computational self-organisation) in cognizing agents, emerging in evolution through interactions of a (living/ cognizing) agent with the environment.
\end{abstract}

\section{Understanding Cognition}

Cognitive science is currently defined as a study of processes of knowledge generation through perception, thinking (reasoning), memory, learning, problem solving, and similar. Thagard (2013) makes an extension of the idea of "thinking" to include emotional experience. This move bridges some of the distance between cognition as thinking and its (sub-)processes, but the fundamental problem of generative mechanisms that can dynamically overarch the chasm between matter and mind remains. The definition of cognitive science does not mention biology, chemistry, (quantum- nano-, etc.) physics or chaos theory, self-organisation, and artificial life, artificial intelligence or data science, extended mind, or distributed cognition as studied with help of network science, sociology or ecology.

On the current view, cognition is about high-level processes remote from physicalchemical-biological substrate. It is modeled either by classical sequential computation, understood as symbol manipulation, or by neural networks. On the other hand, historically, behaviorism offered an alternative view of cognition with the focus on the observable behavior of a subject. This divide is mirrored in the present day schism between cognitivism/computationalism on one side and EEEE (embodied, embedded, enactive, extended) cognition on the other. There have been numerous attempts to bridge this gap 
(Clark 2013), (Scheutz 2002), (Pfeifer and Iida 2005) and others, offering connection between lower level sub-symbolic signal processing and higher-level processes of (classical, mental) cognition.

The most frequent view of cognition is still human-centric and not evolutionary, generative model. Thagard (2014) lists open philosophical problems of this approach to cognition. Majority of those problems can only be solved on the basis of empirical data, experiments and adequate generative models and simulations.

The idea of morphological computing has been proposed by (Paul 2004) (Pfeifer and Iida 2005), (Hauser et al. 2014) and (Müller and Hoffmann 2017) defining computation in a more general way than the traditional symbol manipulation, or connectionist models. It is taking into account physical embodiment of computational mechanisms, thus presenting suitable tool for modeling of a broader range of cognitive phenomena. In a related approach, (Dodig-Crnkovic 2014) takes cognition in a cognitive agent to be morphological computation, defined as information processing performed by morphology on several levels of organization. Cognition in this framework is capacity possessed by all living organisms, as (Maturana and Varela 1980) and (Stewart 1996) argued. Every single cell, while alive, constantly cognizes. It registers inputs from the world and its own body, ensures continuous existence through morphological processes run on metabolic production of energy. It is avoiding dangers that could cause disintegration or damage, adapting its morphology to the environmental constraints. Physicochemical-biological processes present morphological computation on different levels of organization. They depend on the morphology of the organism: its material, form and structure.

Morphological computation is modeled as a dynamics of a structure of nodes (agents) that exchange (communicate) information. Single living cell presents such a structure. Groups of unicellular organisms (such as bacteria) communicate and build swarms or films through morphological computation that presents social/distributed cognition. Groups of cells through morphological computation cluster into multicellular assemblies with specific control mechanisms, forming the tissues, organs, organisms and groups of organisms. This layered organization of networks within networks provides information processing speed-up.

A new quality in morphological computing in living organisms emerges with the development of nervous system. With it, multicellular organisms as cognizing agents acquire ability of self-representation, which enables distinction between "me" and the "other" and presents basic functionality that supports locomotion. Animals that possess nervous systems with centralized control connected to sensors and actuators, are capable of locomotion which increases probability of survival. Brains in animals consist of large number of mutually communicating cells. A single neuron is a relatively simple information processor, while the whole brain possesses advanced information processing/ computational capacities. We see the similar mechanism as in bacteria swarms with distributed cognition implemented as morphological computation.

Besides the ability to model cognition as embodied, embedded, enactive, and extended through interactions with the environment, morphological computing provides means of understanding how this capacity evolved and how it develops during the life of an organism. 


\section{Problems Solutions with a Broader View of Cognition}

Revisiting the list of unsolved/unsolvable problems of cognitive science under the current idea of cognition (Thagard 2014) we can see their natural solution under a more general concept of cognition as morphological computation:

The Emotion Challenge: Morphological computing of embodied cognition has layered computational architecture. Sub-symbolic electro-chemical processes present the basic layer in the information processing related to emotion (von Haugwitz and DodigCrnkovic 2015).

The Consciousness Challenge: Consciousness is proposed as information integration that has central role in the control of behavior (Tononi 2004) (Freeman 2009).

The World Challenge: Distributed morphological computation processes representing hierarchies of computation solves this problem (Abramsky and Coecke 2007) (Sloman 2011) (Piccinini and Shagrir 2014) (Dodig-Crnkovic 2016, 2017).

The Body Challenge: Explicit modeling of a body is a consequence of the inclusion of morphological computational processes in the substrate as an integral part of cognition (Matsushita et al. 2005) (Pfeifer and Bongard 2006) (MacLennan 2010).

The Dynamical Systems Challenge: Dynamical systems are a very important class of computational systems, as argued in (van Leeuwen and Wiedermann 2017) (Burgin and Dodig-Crnkovic 2015).

The Social Challenge: Adopting cognition that is not only individual but also distributed/social, solves this problem (Epstein 2007) (Barabasi 2010).

The Mathematics Challenge (brain cannot be conventional computer): Morphological computing in living beings (unconventional computing) starts at quantum level and propagates to higher levels of organisation as different kinds of physical, chemical, biological, cognitive and social computing. (Cooper 2012) (Zenil 2012).

This short account presents an outline of an argument for the adoption of a broader view of cognition then the one that presents the current received view. For the future work, it remains to study the exact mechanisms of morphological computation at variety of levels of organisation of living organisms in terms of computation as information self-structuring (Dodig-Crnkovic 2016 and 2017). At the same time, cognitive computational models are being tested in artifactual cognitive systems with artificial intelligence and cognitive computing.

\section{References}

Abramsky, S., Coecke, B.: Physics from computer science. Int. J. Unconv. Comput. 3(3), 179197 (2007)

Barabasi, A.-L.: Bursts: The Hidden Pattern Behind Everything We Do. Dutton, London (2010) 
Burgin, M., Dodig-Crnkovic, G.: A taxonomy of computation and information architecture. In: ECSA 2015 ASDS Workshop, Proceedings of the 2015 European Conference on Software Architecture Workshops (ECSAW 2015). ACM, New York (2015)

Clark, A.: Whatever next? Predictive brains, situated agents, and the future of cognitive science. Behav. Brain Sci. 36(3), 181-204 (2013)

Cooper, S.B.: The mathematician's bias and the return to embodied computation. In: Zenil, H. (ed.) A Computable Universe: Understanding and Exploring Nature as Computation. World Scientific Pub. Co Inc. (2012)

Epstein, J.M.: Generative Social Science: Studies in Agent-Based Computational Modeling. Princeton University, Princeton (2007)

Dodig-Crnkovic, G.: Modeling life as cognitive info-computation. In: Beckmann, A., CsuhajVarjú, E., Meer, K. (eds.) Computability in Europe 2014, Proceedings of the 10th Computability in Europe 2014, Language, Life, Limits. LNCS, Budapest, Hungary, 23-27 June 2014. Springer (2014)

Dodig-Crnkovic, G.: Information, computation, cognition. Agency-based hierarchies of levels. (author's draft). In: Müller, V.C. (ed.) Fundamental Issues of Artificial Intelligence. Synthese Library, vol. 377, pp. 139-159. Springer International Publishing, Cham (2016). https:// doi.org/10.1007/978-3-319-26485-1_10, http://arxiv.org/abs/1311.0413

Dodig-Crnkovic, G.: Nature as a network of morphological infocomputational processes for cognitive agents. Eur. Phys. J. Spec. Top. 226, 181-195 (2017). https://doi.org/10.1140/epjst/ e2016-60362-9

Freeman, W.J.: The neurobiological infrastructure of natural computing: intentionality. New Math. Nat. Comput. 5, 19-29 (2009)

Hauser, H., Füchslin, R.M., Pfeifer, R. (eds.): Opinions and Outlooks on Morphological Computation (2014). ISBN (Electronic) 978-3-033-04515-6. http://www.merlin.uzh.ch/ contributionDocument/download/7499. Accessed 28 Jan 2018

MacLennan, B.J.: Morphogenesis as a model for nano communication. Nano Commun. Netw. 1, 199-208 (2010)

Matsushita, K., Lungarella, M., Paul, C., Yokoi, H.: Locomoting with less computation but more morphology. In: Proceedings of the 2005 IEEE International Conference on Robotics and Automation, pp. 2008-2013 (2005)

Maturana, H.R., Varela, F.J.: Autopoiesis and cognition - the realization of the living. In: Cohen, R.S., Wartofsky, M.W. (eds.) Boston Studies in the Philosophy of Science, vol. 42. D. Reidel Publishing, Dordrecht (1980)

Müller, V.C., Hoffmann, M.: What is morphological computation? On how the body contributes to cognition and control. Artif. Life 23, 1-24 (2017)

Paul, C.: Morphology and computation. In: Proceedings of the International Conference on the Simulation of Adaptive Behaviour Los Angeles, CA, USA, pp. 33-38 (2004)

Pfeifer, R., Bongard, J.: How the Body Shapes the Way We Think. A New View of Intelligence. MIT Press, Cambridge (2006)

Pfeifer, R., Iida, F.: Morphological computation: connecting body, brain and environment. Jpn. Sci. Mon. 58(2), 48-54 (2005)

Piccinini, G., Shagrir, O.: Foundations of computational neuroscience. Curr. Opin. Neurobiol. 25, 25-30 (2014)

Scheutz, M. (ed.): Computationalism: New Directions. Cambridge University Press, Cambridge (2002)

Sloman, A.: What's information, for an organism or intelligent machine? How can a machine or organism mean? (Book chapter). In: Dodig-Crnkovic, G., Burgin, M. (eds.) Information and Computation. Series in Information Studies. World Scientific Publishing Co (2011) 
Stewart, J.: Cognition = Life: implications for higher-level cognition. Behav. Proc. 35, 311-326 (1996)

Thagard, P.: Cognitive science. In: Encyclopedia Britannica (2013). https://www.britannica.com/ science/cognitive-science. Accessed 28 Jan 2018

Thagard, P.: Cognitive science. In: Zalta, E.N. (ed.) The Stanford Encyclopedia of Philosophy (Fall 2014 Edition) (2014). https://plato.stanford.edu/archives/fall2014/entries/cognitivescience/. Accessed 28 Jan 2018

Tononi, G.: An information integration theory of consciousness. BMC Neurosci. 5, 42 (2004) van Leeuwen, J., Wiedermann, J.: Knowledge, representation and the dynamics of computation. In: Dodig-Crnkovic, F., Giovagnoli, R. (eds.) Representation and Reality in Humans, Other Living Organisms and Intelligent Machines, p. 69. Springer, Cham (2017)

von Haugwitz, R., Dodig-Crnkovic, G.: Probabilistic computation and emotion as self-regulation. In: ECSA 2015 ASDS Workshop, Proceedings of the 2015 European Conference on Software Architecture Workshops (ECSAW 2015). ACM, New York (2015)

Zenil, H. (ed.): A Computable Universe. Understanding Computation \& Exploring Nature As Computation. World Scientific Publishing Company/Imperial College Press, Singapore (2012) 